JOURNAL OF MODERN OPTICS, 1998, VOL. 45, NO. 9, 1797-1807

\title{
Performance analysis of optical imaging systems based on the fractional Fourier transform
}

\author{
SERGIO GRANIERI*, ENRIQUE E. SICRE \\ Centro de Investigaciones Opticas (CIOp), PO Box 124, 1900 La Plata, \\ Argentina
}

and WALTER D. FURLAN

Departamento de Optica, Universidad de Valencia, 46100 Burjassot, Spain

(Received 2 June 1997; revision received 31 October 1997)

\begin{abstract}
Some image quality parameters, such as the Strehl ratio and the optical transfer function, are analysed in the generalized phase-space, or $x-p$ domain, of the fractional Fourier transform associated with a modified onedimensional pupil function. Some experimental results together with computer simulations are performed which illustrate the tolerance to defocus of different apertures.
\end{abstract}

\section{Introduction}

There are several criteria for analysing the performance of an optical imaging system for aberrations and/or focus errors. Among them we mention: Rayleigh's criterion [1], Maréchal's treatment of tolerance [2] and the Strehl ratio [3]. In these approachs, the on-axis image intensity is the relevant quantity. However, as Hopkins has suggested [4,5], the analysis of Maréchal can be reformulated in order to give a tolerance criterion based on the behaviour of the optical transfer function (spatial frequency information) instead of the point-spread function (space information). More recently, some papers were published in which the Wigner distribution function (WDF) or the ambiguity function (AF) are employed to evaluate image quality parameters [6-12]. This point of view equally emphasizes both the spatial and the spectral information contents of the diffracted wavefields that propagate in the optical imaging systems.

On the other hand, the concept of the fractional Fourier transform (FRT) was introduced into optics by Mendlovic, Ozaktas and Lohmann [13-16]. From a viewpoint based on the WDF, since an ordinary Fourier transform results from a $\pi / 2$ coordinate rotation of the input WDF, the FRT of order $p$ can be achieved through a WDF phase-space rotation by an angle $\phi=p \pi / 2$. Thus, the information content stored in the FR'T changes from purely spatial to purely spectral as $p$ varies from $p=0$ to $p=1$. By taking into account the link between the FRT formalism and the free-space diffraction [17-19], the imaging properties of a given

* Also Facultad de Ciencias Exactas, Universidad Nacional de La Plata, 1900 La Plata, Argentina. 
optical system, both in a space and a spatial frequency domain, can be evaluated from the FRTs of the pupil function for varying fractional order $p$.

In section 2 we discuss some relevant definitions and basic properties of the FRT and the WDF. In section 3 we analyse some image quality parameters, such as the Strehl ratio (SR) or the optical transfer function (OTF), from the FRT of a given pupil aperture. Finally, in section 4 we show some results in order to illustrate the proposed approach.

\section{Basic properties of the FRT}

As was previously mentioned, the FRT of order $p$ can be achieved through a $\mathrm{WDF}$ rotation in the phase-space by an angle $\phi=p \pi / 2$. This operation can be obtained by performing three successive coordinate shearings. Taking into account that WDF shearing in the spatial coordinate means free-space propagation while WDF shearing in the spatial frequency corresponds to lens passage, very simple optical processors can be developed to optically realize the FRT [15, 19, 20]. Figures $1(a)$ and $(b)$ show one of these possible optical arrangements and the effect of this system on the object WDF, respectively. By propagating the light field, the FRT of the input object $t(\xi, \eta)$ is obtained at the output plane as the following Fresnel integral

$$
\begin{aligned}
u_{p}(x, y)= & \Im^{(p)}\{t(\xi, \eta)\} \\
= & \exp \left(\frac{i \pi\left(x^{2}+y^{2}\right)}{\lambda f_{0} \tan \phi}\right) \iint_{-\infty}^{\infty} t(\xi, \eta) \exp \left(\frac{i \pi\left(\xi^{2}+\eta^{2}\right)}{\lambda f_{0} \tan \phi}\right) \\
& \times \exp \left(\frac{-2 \pi i(x \xi+y \eta)}{\lambda f_{0} \sin \phi}\right) \mathrm{d} \xi \mathrm{d} \eta,
\end{aligned}
$$

$f_{0}$ being a free scale parameter. For the sake of simplicity one-dimensional functions are to be considered. It should be noted that the FRT as defined by equation (1) does not hold at values of $p$ so low that the Fresnel approximations involved are no longer valid. However, there are other optical definitions of the FRT which remain valid for $p \rightarrow 0$ (see [13]).

The WDF associated with the object $t(\xi)$, which can be defined as

$$
W_{t}(x, \nu)=\int_{-\infty}^{\infty} t(x+\xi / 2) t^{*}(x-\xi / 2) \exp (-2 \pi i \xi \nu) \mathrm{d} \xi
$$

has several interesting properties that are to be used in this paper; namely

$$
\begin{aligned}
W(x, \nu ; z) & =W_{t}(x-\lambda z \nu, \nu), \\
W^{(+)}(x, \nu) & =W_{t}\left(x, \nu+\frac{x}{\lambda f}\right), \\
W^{(p)}(x, \nu) & =W_{t}(x \cos \phi-\nu \sin \phi, x \sin \phi+\nu \cos \phi), \\
\left|u_{p}(x)\right|^{2} & =\int_{-\infty}^{\infty} W^{(p)}(x, \nu) d \nu .
\end{aligned}
$$

Equations (3a), (3b) and (3c) represent the phase-space coordinate transforms that are applied to the input WDF for the cases of free-space propagation (shearing in the space coordinate), passage through a thin lens (shearing in the spatial frequency), and FR'T (rotation), respectively. Equation $(3 d)$, the spatial frequency 
a)

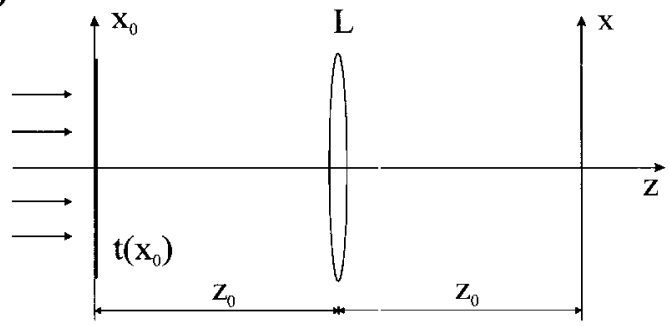

b)

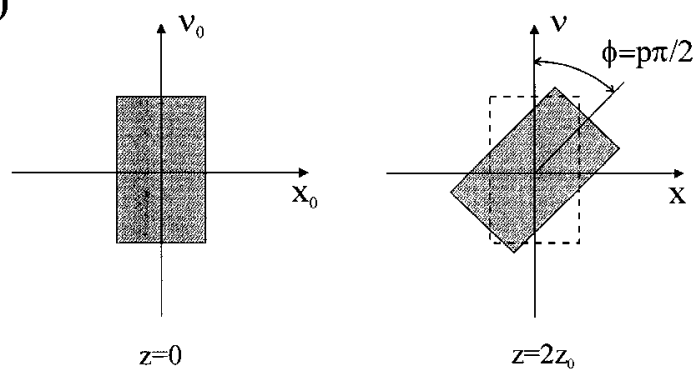

Figure 1. (a) Optical setup to perform the FRT of $t\left(x_{0}\right)$ for a given fractional order $p$, where: $z_{0}=f_{0} \tan (p \pi / 4), f=f_{0} / \sin (p \pi / 2)$ is the focal distance of $L$, and $f_{0}$ is a free scaling factor; $(b)$ Effect of the optical system shown in $(a)$ on the WDF of $t\left(x_{0}\right)$.

projection of the WDF giving rise to the signal intensity, has a practical meaning. As Lohmann and Soffer have demonstrated [21], it also gives the Radon-Wigner transform of the object as the squared modulus of the FRT.

\section{Image quality parameters: a FRT approach}

By referring to figure 2, a normally incident plane wave of unit amplitude illuminates the optical imaging system denoted by $L$. In the neighbourhood of the image plane, located at $z=0$, the field amplitude distribution can be written as

$$
u(x ; z)=\int_{-\infty}^{\infty} T(\xi) \exp \left(\frac{i \pi}{\lambda(f+z)}(\xi-x)^{2}\right) \mathrm{d} \xi,
$$

where

$$
T(\xi)=t_{0}(\xi) \exp \left(\frac{-i \pi}{\lambda f} \xi^{2}\right)
$$

$t_{0}(\xi)$ being the one-dimensional version of a certain amplitude transmission pupil function $t(\xi, \eta)$. By taking into account the properties of the WDF for both, free space light propagation and light passage through a thin lens, the equivalent relationships to that given by equations (4) and (5) can be expressed in terms of the WDF as

$$
\begin{aligned}
W_{u}(x, y ; z) & =W_{T}(x-\lambda(f+z) \nu, \nu) \\
W_{T}(x, \nu) & =W_{t_{0}}\left(x, \nu+\frac{x}{\lambda f}\right),
\end{aligned}
$$

and hence 


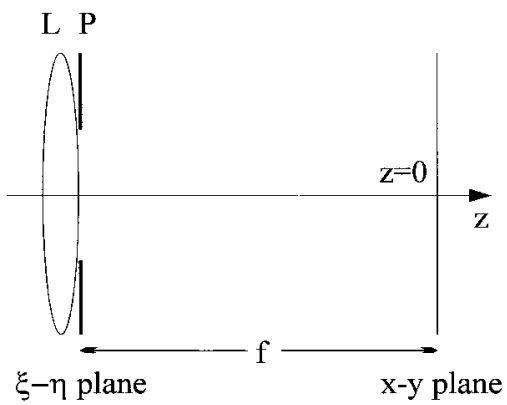

Figure 2. Optical imaging system: the several components are represented by a unique lens $L$, being $P$ the exit pupil.

$$
W_{u}(x, \nu ; z)=W_{t_{0}}\left(x-\lambda(f+z) \nu, \frac{x}{\lambda f}-\frac{z}{f} \nu\right) .
$$

In accordance with equation (8), the on-axis intensity $(x=0)$ for varying $z$ is given by

$$
\begin{aligned}
I(x=0 ; z) & =\int_{-\infty}^{\infty} W(0, \nu ; z) d \nu \\
& =\int_{-\infty}^{\infty} W_{t_{0}}\left(-\lambda(f+z) \nu,-\frac{z}{f} \nu\right) d \nu .
\end{aligned}
$$

For the especial case $z=0$ the intensity can be expressed as

$$
I(x=0, z=0)=\int_{-\infty}^{\infty} W_{t_{0}}(-\lambda f \nu, 0) d \nu .
$$

By making: $\zeta=-z \nu / f$ in equation (9), the Strehl ratio (SR) versus defocus is obtained (for $z \neq 0$ )

$$
\begin{aligned}
S(z) & =I(x=0 ; z) / I(x=0 ; z=0) \\
& =\int_{-\infty}^{\infty} W_{t_{0}}\left(\frac{\lambda f(f+z)}{z} \zeta, \zeta\right) \mathrm{d} \zeta
\end{aligned}
$$

i.e. the SR for variable $z$ can be analysed in a polar fashion in the two-dimensional domain of the WDF associated with the pupil function $t_{0}(\xi)$. The slope of each slice is given by: $\tan \theta=(f+z) / z$.

The imaging properties of the optical system can also be alternatively described in a different phase-space domain by making use of equations (3). The FRT modulus squared of the pupil function results

$$
\left|u_{p}(x)\right|^{2}=\int_{-\infty}^{\infty} W_{t_{0}}\left(x \cos \phi-\nu \sin \phi, x \sin \phi^{+} \nu \cos \phi\right) d \nu .
$$

Equation (12) gives the one-dimensional FRT of $t_{0}(\xi)$ for a certain order $p$, but it can also be considered as the two-dimensional representation of $t_{0}(\xi)$ in a domain: spatial coordinate-fractional order. This signal description is known as the RadonWigner transform (RWT) and some of its properties and possible optical implementations were recently studied [21-23]. Working in the plane $(x, p)$, 
where $\left|u_{p}(x)\right|^{2}$ is displayed as the several FRTs of $t_{0}(\xi)$ (vertical axis) for varying $\operatorname{order} p$ (horizontal axis), we select $x=0$; i.e. we record

$$
\begin{aligned}
\left|u_{p}(0)\right|^{2} & =\int_{-\infty}^{\infty} W_{t_{0}}(-\nu \sin \phi, \nu \cos \phi) d \nu \\
& =(1 / \cos \phi) \int_{-\infty}^{\infty} W_{t_{0}}(-\zeta \tan \phi, \zeta) \mathrm{d} \zeta .
\end{aligned}
$$

By properly normalizing $\left|u_{p}(0)\right|^{2}$, the analogy between equations (11) and (13) is complete whenever

$$
p=\frac{2}{\pi} \arctan \left(\frac{f+z}{z}\right)
$$

and

$$
\left|u_{p}(0)\right|^{2}=S(z(p)) .
$$

Therefore, the values of the SR associated with the pupil function $t_{0}(\xi)$ for different amount of defocus lie on the horizontal axis of the RWT of $t_{0}(\xi)$, between $p=1$ (in-focus situation: $z=0$ ) and $p=0.5$ (maximum out-of-focus plane: $z \rightarrow \infty)$; see figure 3 .

The knowledge of the SR is useful in order to characterize the depth of focus properties of the optical system. The quality of the image itself is better described through the behaviour of the associated OTF. This information can also be obtained from the RWT if we perform a one-dimensional ordinary Fourier transform of $\left|u_{p}(x)\right|^{2}$ along the $x$-axis. By making use of the expression given by equation (1) for the FRT it results in

$$
\begin{aligned}
\Im_{x}\left\{\left|u_{p}(x)\right|^{2}\right\}= & \int_{-\infty}^{\infty} \mathrm{d} x^{\prime} \exp \left(-\frac{2 \pi i x x^{\prime}}{\lambda f}\right) \\
& \times\left|\int_{-\infty}^{\infty} \mathrm{d} \xi t_{0}(\xi) \exp \left(\frac{i \pi \xi^{2}}{\lambda f_{0} \tan \phi}\right) \exp \left(\frac{-2 \pi i x^{\prime} \xi}{\lambda f_{0} \sin \phi}\right)\right|^{2},
\end{aligned}
$$

where $f$ is the focal length of the Fourier transformer. By rearranging the integrals of equation (16), it can be written as

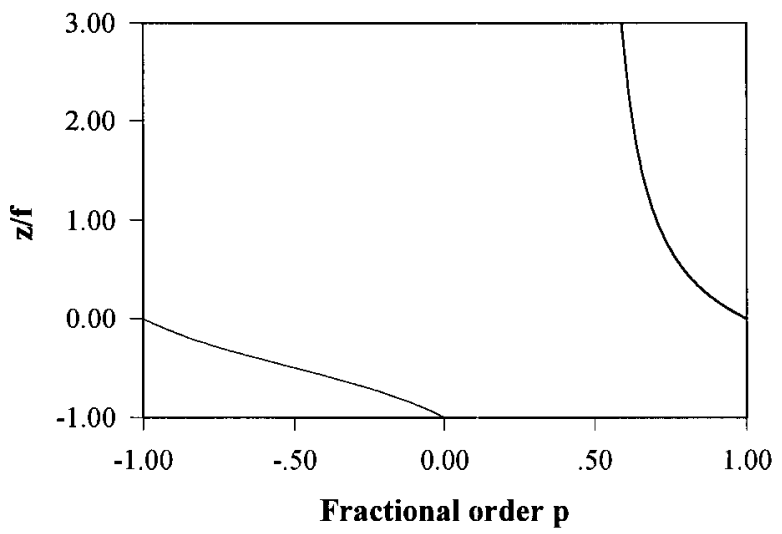

Figure 3. Defocus distance $z$ versus fractional order $p$, as given by equation (10). 


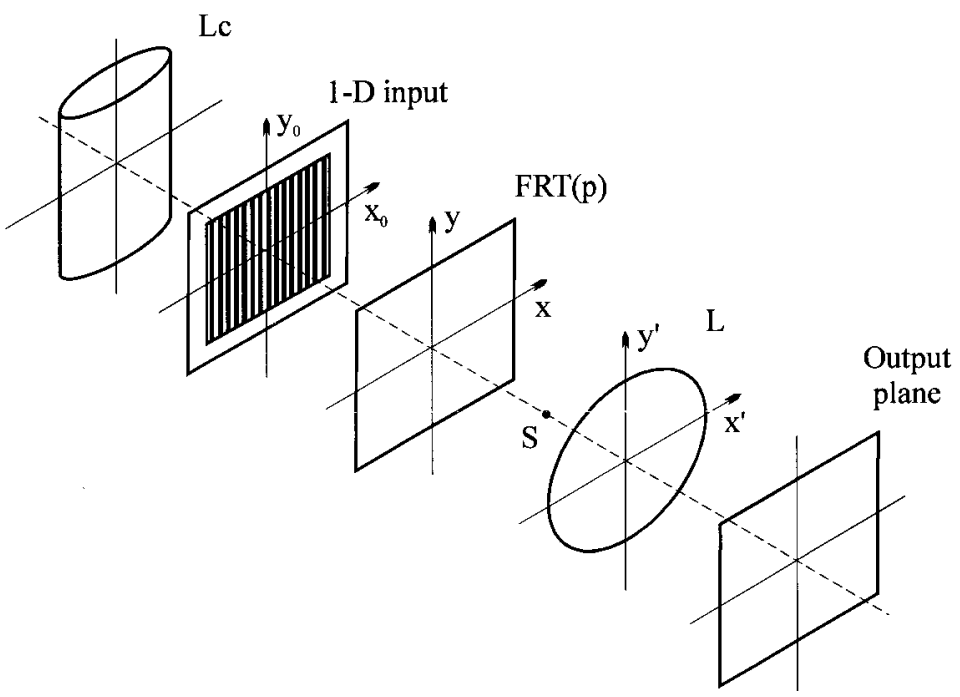

Figure 4. Optical setup for performing the RWT of a one-dimensional input. The cylindrical lens $L_{\mathrm{C}}$ provides the illuminating converging wavefront. The free-space propagation originates the several FRTs at different planes. Each one of such is imaged by the varifocal lens $L$ at the output plane.
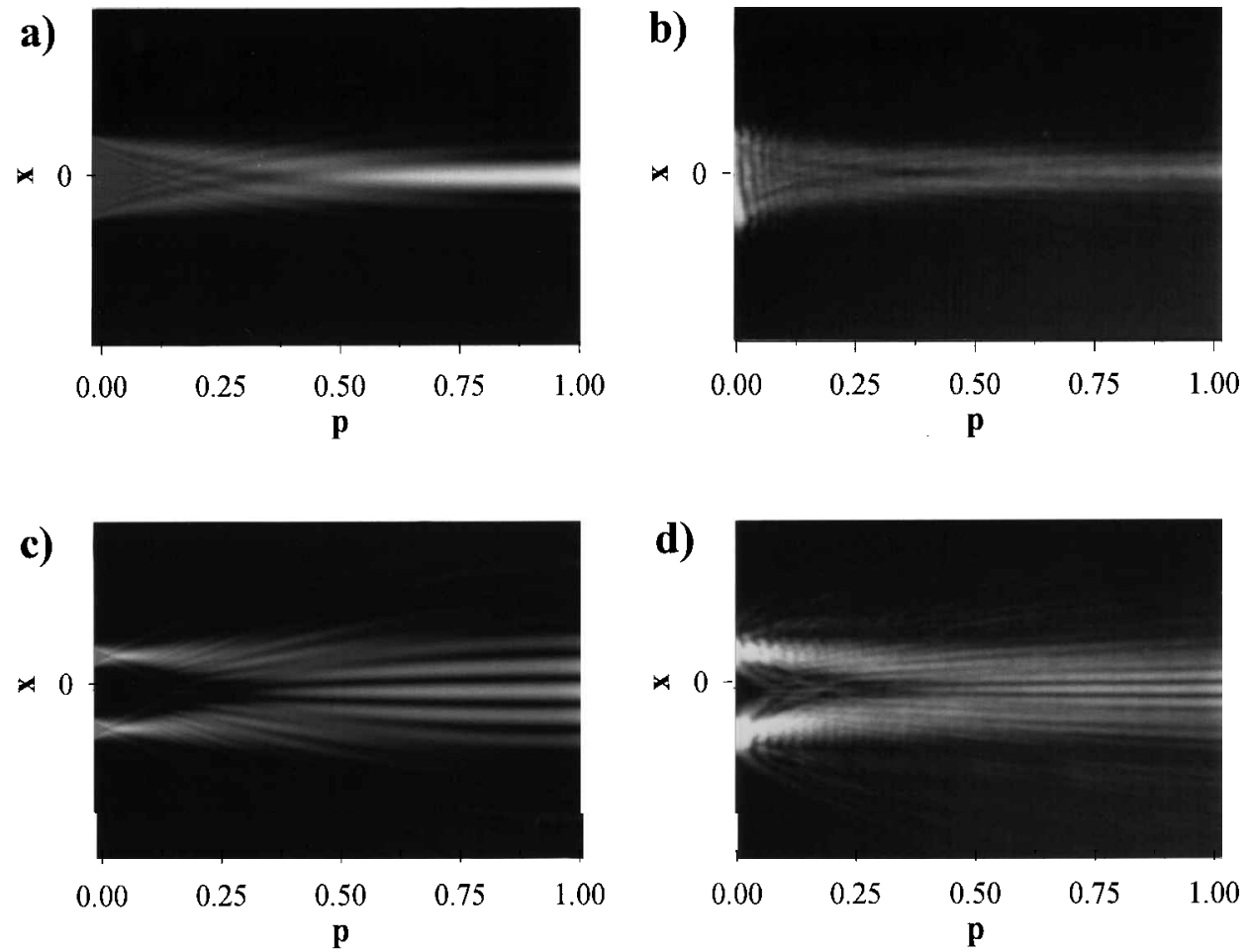

Figure 5. Radon-Wigner transforms: (a) Computer simulation for an aperture with $a=2.5 \mathrm{~mm}$ and $b=0.0 \mathrm{~mm}$; $(b)$ experimental result for the case $(a) ;(c)$ Computer simulation for an aperture with $a=2.5 \mathrm{~mm}$ and $b=1.3 \mathrm{~mm} ;(d)$ experimental result for the case $(c)$. 

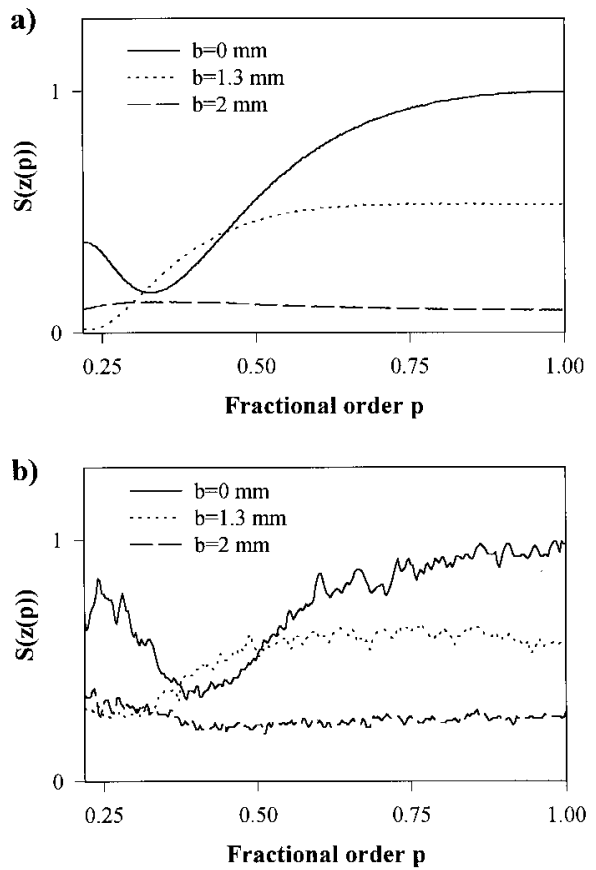

Figure 6. Strehl ratio (SR) versus defocus for the three cases studied.

a)

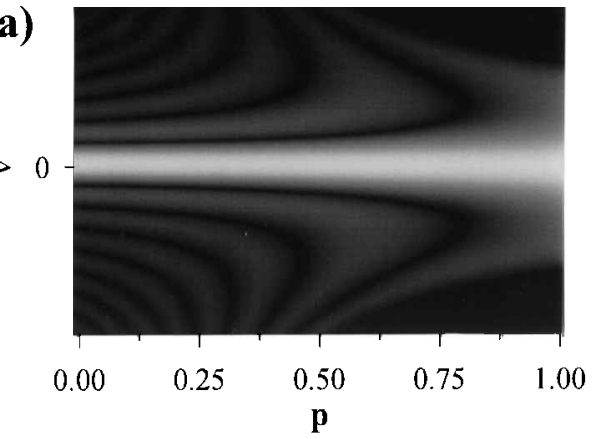

c)

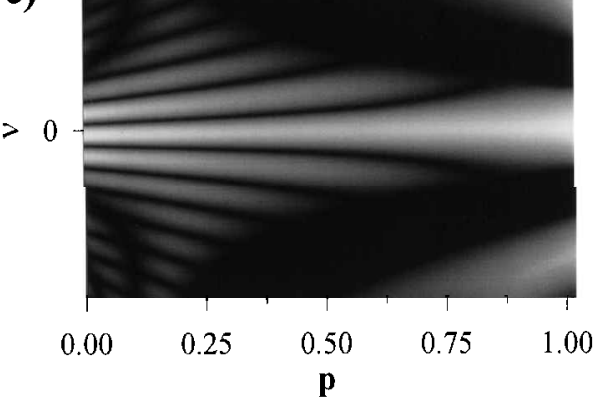

b)

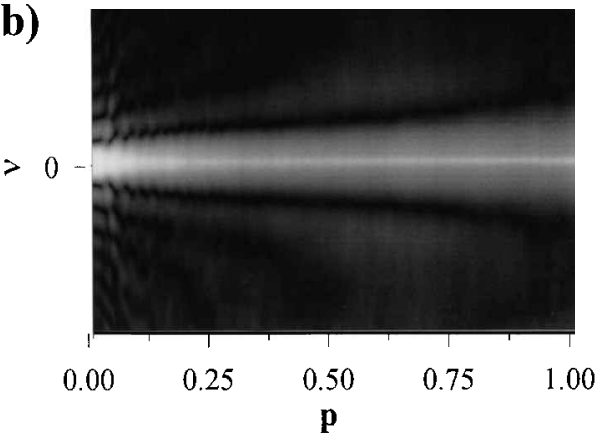

d)

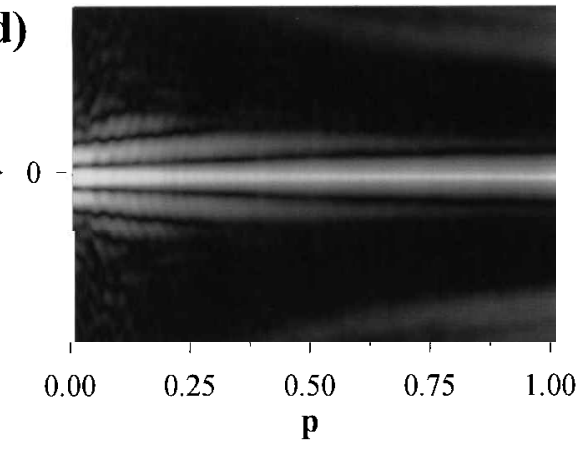

Figure 7. Computed one-dimensional Fourier transforms of the Radon-Wigner transforms shown in figure 5 . 


$$
\begin{aligned}
\Im_{x}\left\{\left|u_{p}(x)\right|^{2}\right\} & =\int_{-\infty}^{\infty} \mathrm{d} \xi^{\prime} t_{0}\left(\xi^{\prime}+\frac{f_{0} x \sin \phi}{2 f}\right) t_{0}^{*}\left(\xi^{\prime}-\frac{f_{0} x \sin \phi}{2 f}\right) \exp \left(\frac{2 \pi i \cos \phi x \xi^{\prime}}{\lambda f}\right) \\
& =A_{t_{0}}\left(\frac{f_{0} x \sin \phi}{f}, \frac{x \cos \phi}{\lambda f}\right),
\end{aligned}
$$

$A_{t_{0}}(\mu, y)$ being the ambiguity function (AF) which is also a phase-space representation of $t_{0}(\xi)$. Since the AF simultaneously contains all the OTFs associated with the optical system with varying focus errors according to the formula [6]

$$
\mathcal{H}\left(\nu ; w_{20}\right)=A\left(\nu, y=\frac{2 w_{20 \nu}}{\lambda \nu_{0}^{2}}\right),
$$

$w_{20}$ being the wavefront focus error coefficient and $\nu_{0}$ the cutoff spatial frequency, equation (17) becomes

$$
\Im_{x}\left\{\left|u_{p}(x)\right|^{2}\right\}=\mathcal{H}\left(\nu ; w_{20}=\frac{\nu_{0}^{2} \cot (p \pi / 2)}{2 f_{0}}\right) .
$$

In this way, for a given value of the fractional order $p$ (along the horizontal axis) there is a defocused OTF displayed along the vertical or spatial frequency axis. This representation is quite convenient to visualize the Hopkins's criterion [7].
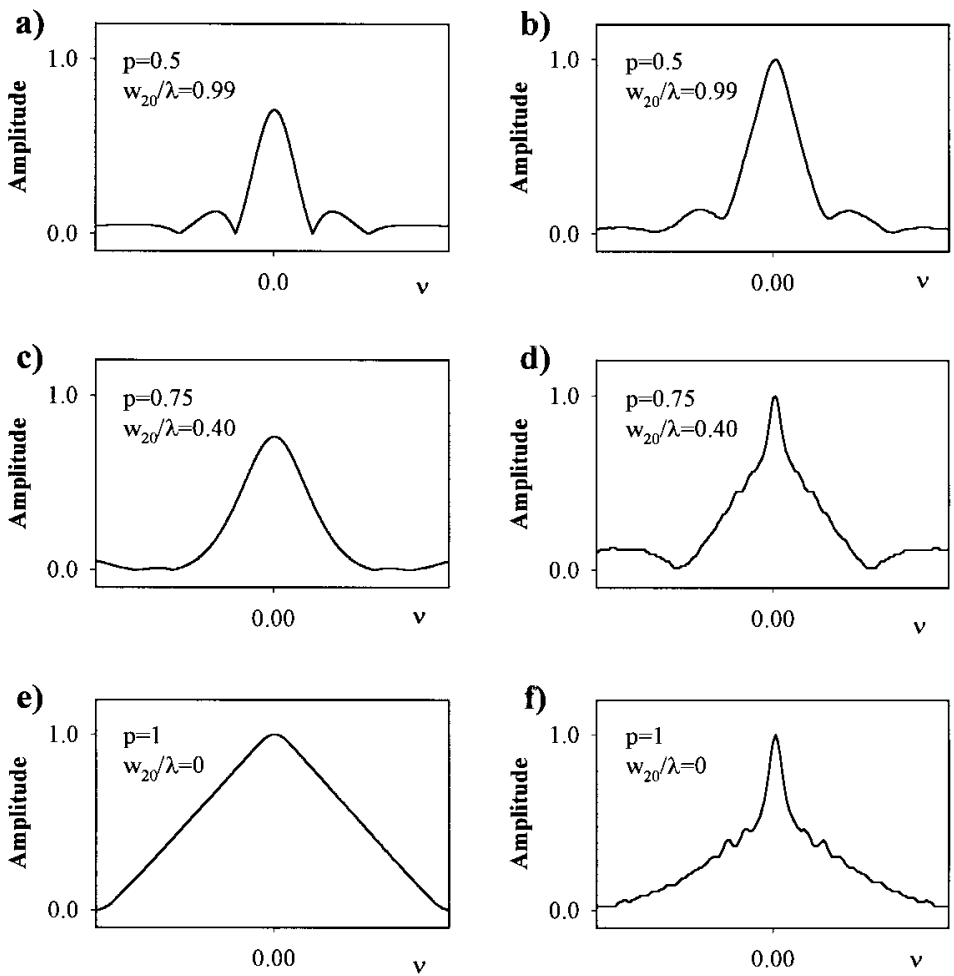

Figure 8. Optical transfer functions obtained from different slices of the intensity distributions shown in figure $7(a)((a),(c)$ and $(e))$ and figure $7(b)((b),(d)$ and $(f))$, for the case of a uniform aperture. 

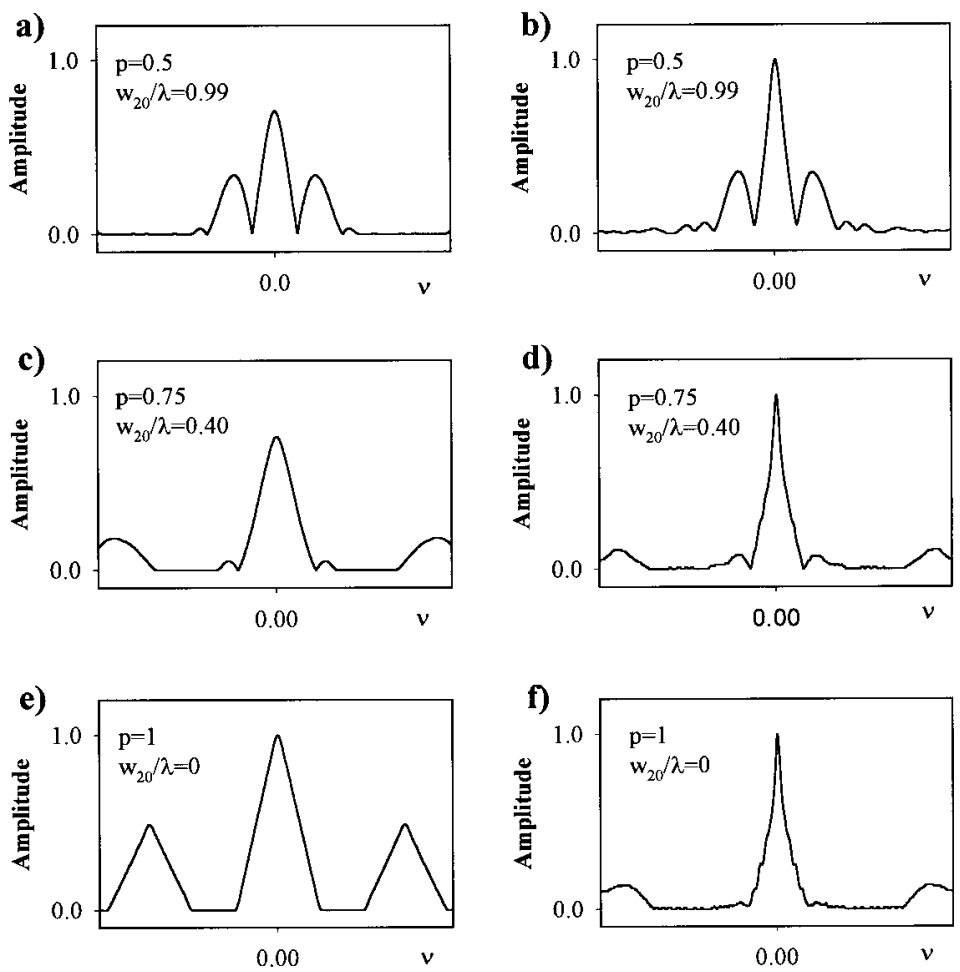

Figure 9. Optical transfer functions obtained from different slices of the intensity distributions shown in figure $7(c)((a),(c)$ and $(e))$ and figure $7(d)((b),(d)$ and $(f))$, for the case of an annular aperture.

\section{Experimental results}

The optical setup employed for obtaining the RW'T of a given one-dimensional transmittance is sketched in figure 4. As was discussed in [22], a cylindrical convergent wave illuminating the input object gives rise by free-space diffraction to the different FRTs with varying order $p$. These FRTs are located at different planes with different lateral magnifications. The action of a varifocal lens $L$ is to image the FRTs in a unique output plane with (approximately) the same magnification. The horizontal $x$-axis displays the FRT for each value of $p$ obtained along the vertical $y$-axis. Thus, the intensity distribution at this plane becomes the RWT.

In order to illustrate the approach developed in section 3, we compare the tolerance to defocus of a transparent but finite size aperture and a pupil with a central obscuration. As in equation (4), the modified one-dimensional pupil $t_{0}(\xi)$ becomes

$$
t_{0}(\xi)=\operatorname{rect}(\xi / a)-\operatorname{rect}(\xi / b), \quad b \leq a
$$

and three cases are considered: (i) uniform aperture, $b=0$; (ii) annular aperture, with $b=0.52 a$; and (iii) annular aperture, with $b=0.80 a$. Figure 5 shows the RWTs numerically ( $a$ and $c$ ) and experimentally ( $b$ and $d$ ) obtained, for the cases (i) and (ii). Accordingly with equations (14) and (15), the slices of the RWT for $x=0$ give rise to the SR for variable $z$, which are plotted in figure 6 for the three 
cases analysed. From these results, it can be observed the higher tolerance to defocus of the annular apertures as should be expected.

Figure 7 shows the one-dimensional Fourier transforms, taken with respect to the $x$-axis (see figure 4), of the RWT illustrated in figure 5. From the analysis carried out in section 3 to derive equation (19), the defocused OTFs are displayed along the vertical or spatial-frequency axis. These results are shown in figures 8 and 9, for the pupils (i) and (ii), respectively, and for: $p=0.5\left(w_{20} / \lambda=0.99\right)$, $p=0.75\left(w_{20} / \lambda=0.40\right)$, and $p=1\left(w_{20} / \lambda=0\right)$.

\section{Conclusions}

We have applied the FRT formalism for analysing the performance of some optical imaging systems. This approach can be considered as a generalization of those based on phase-space signal representations, such as the WDF or the AF. The information about the image quality parameters is stored in the FRT modulus squared, or RWT, associated with a one-dimensional transmittance. This one can be considered as a modified pupil function of an equivalent two-dimensional symmetric pupil aperture. The intensity distribution along a slice of this RWT allows one to obtain the SR versus defocus, while performing a Fourier transformation along one axis gives rise to a display of the different defocused OTFs. In order to illustrate this approach we compared the tolerance to defocus of a uniform pupil and a pupil with a central obscuration.

\section{Acknowledgments}

Financial support from Agencia Nacional de Promoción Científica y Tecnológica, SECYT (Argentina), Project PMT-PICT 0289 is gratefully acknowledged. Walter D. Furlan acknowledges financial support from the Dirección General de Investigación Científica y Técnica (Spain) (Grant PB93-0354-C02-01).

\section{References}

[1] Lord Rayleigh, 1879, Phil. Mag., 8, 261.

[2] Maréchal, A., 1947, Rev. Opt., 26, 257.

[3] Strehl, K., 1902, Zeitfür Instrumkde, 22, 213.

[4] Hopkins, H. H., 1957, Proc. Phys. Soc. London B, 70, 449.

[5] Hopkins, H. H., 1966, Optica Acta, 13, 343.

[6] Brenner, K.-H., Lohmann, A. W., and Ojeda-Castañeda, J., 1983, Optics Commun., 44, 323.

[7] Bartelt, H., Ojeda-Castañeda, J., and Sicre, E. E., 1984, Appl. Optics, 23, 2693.

[8] Ojeda-Castañeda, J., Berriel-Valdos, L. R., and Montes, E., 1988, Appl. Optics, 27, 790.

[9] Ojeda-Castañeda, J., Andrés, P., and Montes, E., 1987, J. opt. Soc. Am. A, 4, 313.

[10] Bourimborde, L. V., Furlan, W. D., and Sicre, E. E., 1991, J. mod. Optics, 38, 1685.

[11] Furlan, W. D., Saavedra, G., and Lancis, J., 1993, Optics Commun., 96, 208.

[12] Zalvidea, D., Lehman, M., Granieri, S., and Sicre, E. E., 1995, Optics Commun., 118, 207.

[13] Ozaktas, H. M., and Mendlovic, D., 1993, Optics Commun., 101, 163.

[14] Mendlovic, D., and Ozaktas, H. M., 1993, J. opt. Soc. Am. A, 10, 1875.

[15] Lohmann, A. W., 1993, J. opt. Soc. Am. A, 10, 2181.

[16] Mendlovic, D., Ozaktas, H. M., and Lohmann, A. W., 1994, Appl. Optics, 33, 6182.

[17] Pellat-Finet, P., 1994, Optics Lett., 19, 1388.

[18] Granieri, S, Trabocchi, O., and Sicre, E. E., 1995, Optics Commun., 119, 275. 
[19] Andrés, P., Furlan, W. D., Saavedra, G., and Lohmann, A. W., 1997, J. opt. Soc. Am. A, 14, 853.

[20] Bernardo, L. M., and Soares, O. D. D., 1994, Optics Commun., 110, 517.

[21] Lohmann, A. W., and Soer, B. H., 1994, J. opt. Soc. Am. A, 11, 1798.

[22] Mendlovic, D., Dorsch, A. W., Lohmann, A. W., Zalevsky, Z., and Ferreira C., 1996, Appl. Optics, 35, 3925.

[23] Granieri, S., Furlan, W. D., Saavedra, G., and Andrés P., 1997, Appl. Optics, 36, 8363. 
Copyright of Journal of Modern Optics is the property of Taylor \& Francis Ltd and its content may not be copied or emailed to multiple sites or posted to a listserv without the copyright holder's express written permission. However, users may print, download, or email articles for individual use. 